\title{
The Challenge of Mapping COVID-19 Data
}

\section{Menno-Jan Kraak}

This perspective discusses the design choices to make while dealing with multivariate COVID-19 data; the most common errors made in this process; and how to avoid them and present data in meaningful and effective ways.

\subsection{The Mapping Challenge}

In reporting about the COVID-19 pandemic, many plots, maps and other diagrams showing the current situation at local and global levels and comparing regional developments have appeared in the media.

The complexity of these graphics varies both in design and technology. You can find ingenious online story maps and simple single variable maps in the print media. The data behind the graphics is most often related to three different variables: the infected, the hospitalized and the dead. It is quite a cartographic challenge to visualize all these numbers properly, especially together. This because there is a wide variety over space and time, and a large range between the lowest and highest values, as well as between and within these variables.

The numbers available can be very revealing but also should be interpreted with caution. It is revealing because when plotted over time they show where the pandemic started and how it spread over the world. Caution is needed because countries do not necessarily have the same definition for each variable nor follow the same data collection approach. Some publish everything while others virtually nothing. Some numbers only include those tested while other also those suspected. Numbers after a weekend tend to be higher because most weekend cases are reported only after the weekend. Reading this you might wonder how do we then create trustworthy maps? My advise is that we look at maps critically and realize that there might be a data problem.

\subsection{How-to}

Here I would like to address two issues:

1. professional cartographic design challenges and choices; and

2. the mapmakers problem (or the most common cartographic mistakes in the media).

Let us discuss the challenges based on variations of three familiar questions: where? what? and when? At what spatial scale do we map? Like nearly all phenomena, a pandemic is not 
homogeneously distributed over space. In some places one would prefer a larger scale than in other locations. In addition, the collecting of the numbers does not necessarily happen at the same level of geographical units, and even if so, these might vary in size quite a lot. Inset maps for the more crowded areas could be useful.

How do we symbolize the numbers? The large range between the lowest and highest values is often a delicate design problem. How to select symbol sizes that avoid the big symbols covering the whole map, but still allow us to see the smallest values? This problem gets amplified when all high values are in the same region. One solution is to classify the data, and group individual values together according to a formula. The choice of a classification method is critical because you want to avoid hiding existing patterns or reveal non-existing patterns. It would also be possible to apply logarithmic scales, but one can wonder if users (esp. the public) can easily understand and interpret this. Yet another commonly applied alternative is to normalize the data, for instance display the number of infected per hundred thousand inhabitants.

How do we visualize the trends over time? Similar to the non-homogeneous distribution over space the course of the pandemic over time is also erratic. The depiction of time can be done in a single map, but more often is done in a set of maps or even by animation. Often dashboard like views are used, allowing the display of multiple perspectives with maps and additional time-line diagrams.

\subsection{Case in Point}

A final design will be based on a combination of the above considerations with the overall purpose of the map in mind. In Figure 34.1, we see a detail of a COVID-19 related map of Europe providing data on country level. The map background supports the theme, and shows roads, airports and harbors, the human infrastructure potentially used by the virus to enter a country. The red half circles represent the number of deaths, and the blue halve circles the number of infected. Both half circles have a different scale because the number of infected is a magnitude bigger than the number of deaths. This stresses the importance of a clear map legend. To avoid that the large circles covers too much map, they have been made transparent above a certain size, as for instance can be seen for France and Germany. More information on this map's design choices, can be found in [1]. Following this lead, you can also download this and other maps.

From a cartographic domain perspective, it is great to see so many maps on COVID-19 in the news media. However, despite the creativity of the mapmakers, bad design choices have been made. The most common error is the choice of the wrong map type, especially when the mapmakers try to visualize absolute numbers. Some just plot the number as text in the geographic unit, but such maps have to be literally read unit by unit and will not provide an overview. The 'worst' mistake is when, instead of a map with proportional point symbols like in the map in Figure 1, a choropleth map is used. This map type is only suitable for relative data. The problem is that two geographical units in the same class could differ substantially in size and this results in an over attention for the large unit. Also, the overall pattern might be misleading. Often these choices are due to the default options in the software used.

When choropleths are correctly used, for instance, to display normalized data, such as the number of infected per hundred thousand inhabitants the choice of a map projections is also critical. Since people use these maps to discover patterns and compare regions, only so-called equal area projections should be used. A common error is the use of the Mercator projection which disproportionally exaggerates the size of land masses towards the north and south pole. Its use is - again - often driven by the default options in the software used. 


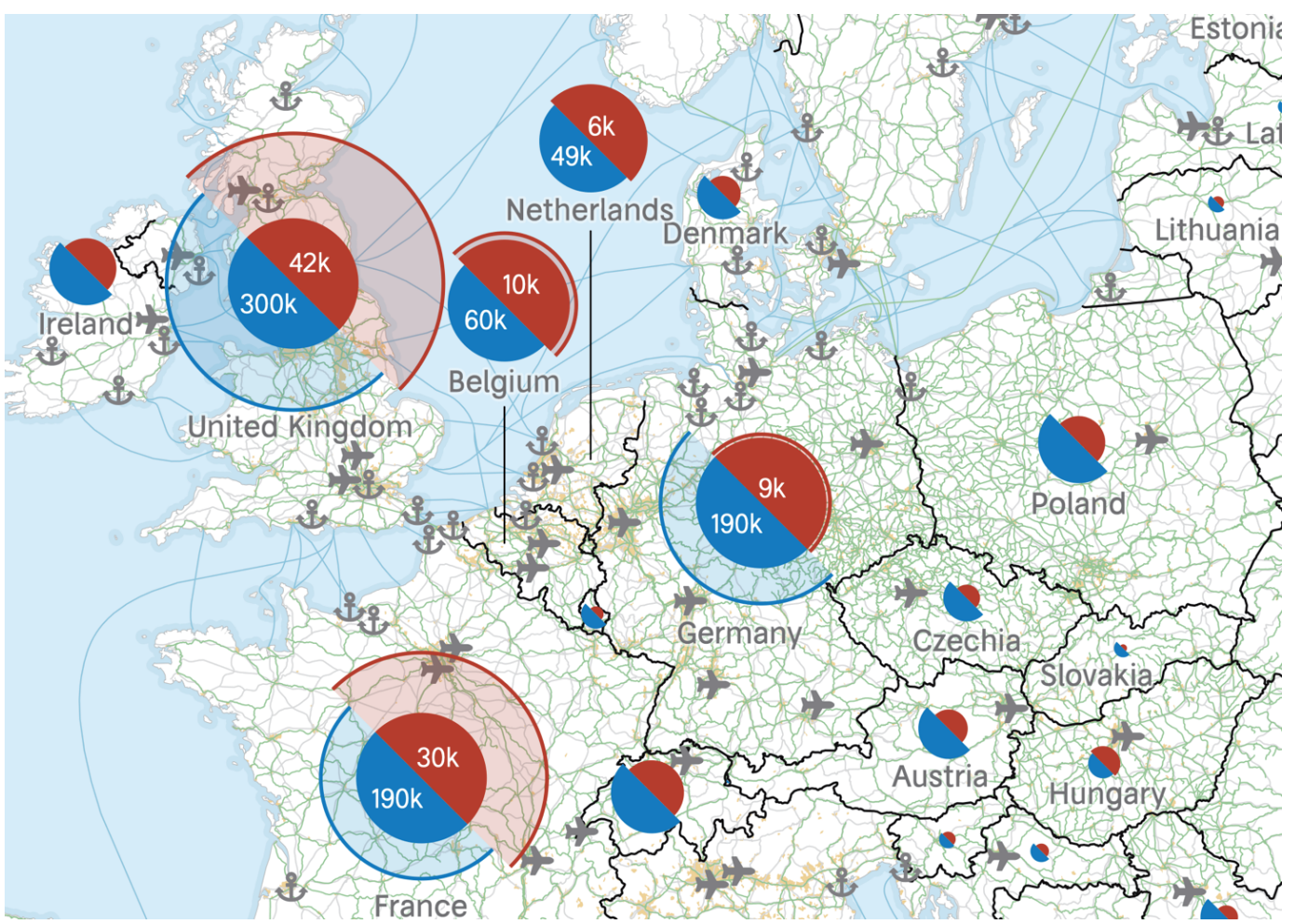

\section{FIGURE 34.1}

Detail of a map about the COVID-19 pandemic in Europe

\subsection{From Data to Insights... to Actions}

The dynamics and variability of the data related to pandemics such as COVID-19 is a real challenge for cartographers and requires creative but critical thinking about solutions which are also influenced by the final environment where the maps are published. Well-designed maps will attract attention and enlighten, providing insights that tell the story at hand and lead to appropriate decisions and actions.

\section{References}

[1] Franz-Benjamin Mocnik, Paulo Raposo, Wim Feringa, Menno-Jan Kraak, and Barend Köbben. Epidemics and pandemics in maps - the case of covid-19. Journal of Maps, 16(1):144-152, 2020. doi: 10.1080/17445647.2020. 1776646. URL https://doi.org/10.1080/17445647.2020.1776646. 
$\Longrightarrow$ Taylor \& Francis Taylor \& Francis Group

http://taylorandfrancis.com 